\title{
Cerebellopontine Angle Epidermoid Cyst: Case Report
}

\author{
Fabio Di Giustino, Rudi Pecci, Beatrice Giannoni, Paolo Vannucchi \\ Department of Surgical Sciences Oto-Neuro-Ophthalmology, Service of Audiology, University of Florence, Florence, Italy \\ Email: fabiodigiustino@libero.it
}

Received October 7, 2012; received November 16, 2012; accepted November 27, 2012

\begin{abstract}
Epidermoid cysts are rare congenital tumors of the central nervous system (CNS), histologically benign and slowgrowing lesions. Their frequency among primitive intracranial tumors is about $1 \%$ and they account for $40 \%$ of all intracranial epidermoid of the cerebellopontine angle (CPA); there they constitute the third most frequent neoplasm (5\%), after acoustic neuromas and meningiomas. We report the case of a patient with a paucisymptomatic epidermoid cyst of the CPA.
\end{abstract}

Keywords: Primary Brain Tumor; Epidermoid Cyst; Cerebellopontine Angle; Stapedius Reflex; Gaze-Evoked Nystagmus

\section{Introduction}

Epidermoid cysts are congenital, rare, slow-growing, benign lesions of the CNS, that arise from ectopic inclusion of ectodermal cells during closure of the neural tube between the third and the fifth weeks of embryonic life $[1,2,4]$. They have a central core of keratin proteins, desquamating cells and cholesterol, lined with a stratified squamous epithelium [3]. They account for $1 \%$ of all intracranial tumors [1-3]; about $40 \%$ of them are located in the CPA, representing the third most frequent lesion, after acoustic neuromas and meningiomas [1,4]. Rarely they are located in the parasellar region, petrous apex, chiasmal region, brainstem and intraventricular cavity [2]. Epidermoid cysts spread along pathways of least resistance such as natural cleavage planes and anatomic canals, extend into more than one cranial fossa and envelop neural and vascular structures. Clinically these tumors produce an insidious and protracted symptoms and signs, with slow growth of the mass, which involves cranial nerves, cerebellar and brainstem structures. The onset of syptoms occurs between the second and fifth decades of life. Common presentations include a long history of tinnitus and hearing loss; vestibular symptoms are seldom seen, and occasionally, symptoms of trigeminal neuralgia, facial paresis or hemifacial spasm, headache, hydrocephalus and chemical or aseptic meningitis occur. The latter can result in rare cases of rupture of the epidermoid lining, so that keratin debris may spill into the subarachnoid space directly causing irritation on arachnoid membranes and nerve parenchymal [5].

Moreover, the symptomatology is different depending on the location of the tumor. In most of the patients with
CPA cysts the first symptoms are subjective (tinnitus, headache, facial paresthesia) and/or functional (trigeminal neuralgia, hemifacial spasm), rather than objective neurological focal impairments. In patients with epidermoids of the parasellar region the clinical onset may be different, presenting with diplopia and seizure (a similar clinical picture may be seen in epidermoids extending into the suprasellar region). Parasellar extended cysts tend to express supratentorial symptoms, whereas mesencephalic extended epidermoids are characterized by higher disturbance rate, especially regarding the brainstem. Posterior fossa basal tumors usually manifest with seizure, whereas headache and gait ataxia are commonly observed in fourth ventricle epidermoids [6].

Diagnosis is based on MRI; images depend on the presence of lipids, cholesterol and keratin [4]. Usually, this lesions display hypointensity on T1-weighted images, with no gadolinium enhancement. T2-weighted images show a nonhomogeneous highsignal-intensity lesion; this kind of images can best define the full extent of the lesion, demonstrating any associated edema. Epidermoid cysts are constituted by a thin capsule with fine internal strands, which may surround rather than displace neurovascular structures [5]. Diffusion-weighted images show bright hyperintensity relative to the brain tissue and cerebrospinal fluid (CSF). FLAIR sequences have a better resolution capable of differentiating between non-free water-like cystic intracranial lesion and CSF [7]. CT scans show a nonenhancing, hypodense lesion.

Epidermoids cysts are not sensitive to radiation or chemotherapy; the treatment relies exclusively on surgical excision, which depends on the location, size, structures involved and symptoms associated. If the tumors 
are located in the CPA, the common approach is through a retrosigmoid incision and suboccipital craniectomy; in cases with supratentorial extension another approach is a subtemporal-transtemporal route; in cases with both supratentorial and infratentorial components, a combined suboccipital and subtemporal approach in one or two stages may be performed.

The aim of surgery is complete removal of the lesion without damage to adjacent neurovascular structures; sometimes portions of the capsule adherent to these structures make this extremely difficult. Some authors advocated a radical excision of the lesion to prevent recurrence, others promote a more conservative approach to minimize morbidity and mortality. Generally it is required more than 4 to 5 years of follow-up to assess whether or not there is recurrence, because of the slowgrowing of these tumors [8].

Incentives for surgery are young age, evidence of tumor growth on diagnostic imaging, and invalidating symptoms; a more conservative attitude could be adopted for older patients (because of the slow growth of the mass and higher surgical risks) and incidental finding without clinical symptoms. The decision not to operate requires necessarily an imaging follow-up, and surgery should be reconsidered for a patient with tumor growth [9]. Generally, the mean duration from onset of symptoms to time of surgery varies between 2 and 5 years [8].

Malignant degeneration is rare. The mechanism of this transformation is unclear; it could depend on chronic inflammation that causes spontaneous rupture and leakage of the cyst contents. The growth rate of epidermoids is linear rather than exponential, as malignant tumors. The incomplete removal of the capsule of the epidermoids may determine the recurrence and/or malignant transformation; in this case subtotal resection and adjuvant therapy are the optimal management strategy. It has been demonstrated that radiosurgery is effective to control malignant epidermoid tumors [10].

\section{Case Report}

A 23-year-old man presented with sudden tinnitus in right ear, ticking-like, accompanied by dizziness and shaking, lasting for 3 minutes. Within few hours, the same symptoms recurred twice. Patient did not have hearing loss. Previously he referred only brief tinnitus and fullness in right ear.

Audiometry revealed bilateral normal hearing, but he did not show stapedius reflex bilaterally on $4 \mathrm{kHz}$ for stimulation of right ear. Vestibular examination showed bilateral gaze-evoked nystagmus and auditory brainstem response (ABR) displayed absence of the waves III-IV-V stimulating right ear. VEMP tests were normal bilaterally. MRI scan (Figure 1) showed a space occupying lesion on the right CPA with enlargement of peripontine space

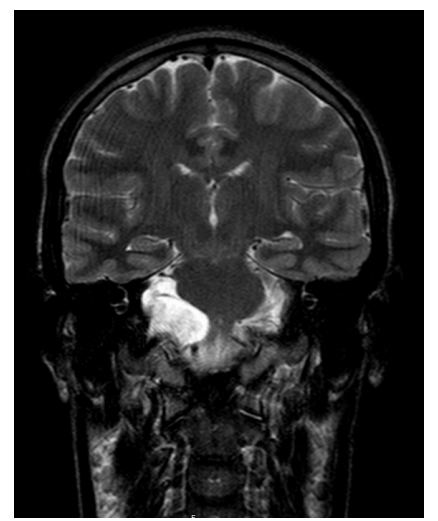

Figure 1. MRI T2-weighted image shows the highsignalintensity lesion on the right CPA.

and without enhancement after injection of gadolinium. CT scan confirmed the presence of a lesion located at the posterior edge of the right temporal pyramid, with a body-effect on the medulla and ponto-medullary junction and a density superior to CSF. This lesion was identified as epidermoid cyst, with a size of $23 \times 27 \times 21 \mathrm{~mm}$ and no growth at following controls. Nowadays the patient still has brief episodes of tinnitus in right ear and dizziness.

\section{Discussion}

Epidermoid cysts are benign tumors with slow growth that tend to envelop neurovascular structure without displacement. Symptoms depend on compression on these structures; often they cause trigeminal neuralgia or facial paresis if fifth and seventh cranial nerves are involved. In other cases the eighth nerve involvement determines poor symptomatology because the slow growth does not cause vertigo, thanks to the compensation capability of the vestibular system. Audiological symptomatology can be poor. Indeed our patient has an inconstant tinnitus in the side of the lesion with brief sensation of dizziness. This symptomatology and the absence of stapedius reflex only for a frequency required other tests such as ABR and MRI that established the diagnosis. However normal caloric tests and cervical VEMPs showed the integrity of vestibulo-ocular and sacculo-collic reflexes. The patient is controlled with MRI every 6 months, because the size of lesion and symptomatology do not suggest a surgical approach. The MRI allows to carefully check the exact growth rate of the lesion and to adopt the "wait-and-see" approach without any risk. This technique is essential during follow-up, especially when surgery is not the first and immediate choice, since MRI is highly specific, sensitive and avoids radiation for the patient [11].

\section{Conclusion}

An accurate evaluation of symptoms and instrumental 
exams permits us to reach the correct diagnosis. We believe that even the only absence of stapedius reflex on one frequency, unilateral, in a young and normoacusic patient should lead to further diagnostic examinations. The same patient had a brief balance disorder of small entity, but which hid an important sign from a topodiagnostic point of view; hence the importance of performing even an otoneurologic examination using videooculoscopy, despite the absence of significant vertiginous symptoms. Indeed, the gaze-evoked nystagmus indicated a lesion on medial vestibular nucleus, hypoglossal preposite nucleus or cerebellar flocculus. Moreover, ABR suggested a CPA lesion for the absence of waves III-IV-V with a normal audiometry. In conclusion, we want to stress the importance of anamnesis and interpretation of clinical and instrumental exams to get a correct diagnosis.

\section{REFERENCES}

[1] D. W. Son, C. H. Choi and S. H. Cha, "Epidermoid Tumors in the Cerebellopontine Angle Presenting with Trigeminal Neuralgia," Journal of Korean Neurosurgical Society, Vol. 47, No. 4, 2010, pp. 271-277. doi:10.3340/jkns.2010.47.4.271

[2] C. K. Chu, H. M. Tseng and Y. H. Young, "Clinical Presentation of Posterior Fossa Epidermoid Cysts," European Archives of Otorhinolaryngology, Vol. 263, No. 6, 2006, pp. 548-551. doi:10.1007/s00405-005-0005-7

[3] A. Akhavan-Sigari, M. Bellinzona, H. Becker and M. Samii, "Epidermoid Cysts of the Cerebellopontine Angle with Extension into the Middle and Anterior Cranial Fossae: Surgical Strategy and Review of the Literature," Acta Neurochirurgica, Vol. 149, No. 4, 2007, pp. 429-432. doi:10.1007/s00701-007-1117-1
[4] E. A. David and J. M. Chen, "Posterior Fossa Epidermoid Cyst,” Otology \& Neurotology, Vol. 24, No. 4, 2003, pp. 699-700. doi:10.1097/00129492-200307000-00028

[5] S. N. Dutt, S. Mirza, S. V. Chavda and R. M. Irving, "Radiologic Differentiation of Intracranial Epidermoids from Arachnoid Cysts,” Otology \& Neurotology, Vol. 23, No. 1, 2002, pp. 84-92. doi:10.1097/00129492-200201000-00019

[6] A. Talacchi, F. Sala, F. Alessandrini, S. Turazzi and A. Bricolo, "Assessment and Surgical Management of Posterior Fossa Epidermoid Tumors: Report of 28 Cases," Neurosurgery, Vol. 42, No. 2, 1998, pp. 242-251. doi:10.1097/00006123-199802000-00020

[7] P. Liu, Y. Saida, H. Yoshioka and Y. Itai, "MR Imaging of Epidermoids at the Cerebellopontine Angle," Magnetic Resonance in Medical Sciences, Vol. 2, No. 3, 2003, pp. 109-115. doi:10.2463/mrms.2.109

[8] T. K. Schiefer and M. J. Link, "Epidermoids of the Cerebellopontine Angle: A 20-Year Experience,” Surgical Neurology, Vol. 70, No. 6, 2008, pp. 584-590. doi:10.1016/j.surneu.2007.12.021

[9] M. Vinchon, B. Pertuzon, J.-P. Lejeune, R. Assaker, J.-P. Pruvo and J.-L. Christiaens, "Intradural Epidermoid Cysts of the Cerebellopontine Angle: Diagnosis and Surgery," Neurosurgery, Vol. 36, No. 1, 1995, pp. 52-57. doi:10.1227/00006123-199501000-00006

[10] S. Hao, J. Tang, Z. Wu, L. Zhang, J. Zhang and Z. Wang, "Natural Malignant Transformation of an Intracranial Epidermoid Cyst," Journal of the Formosan Medical Association, Vol. 109, No. 5, 2010, pp. 390-396. doi:10.1227/00006123-199501000-00006

[11] L. Di Rienzo, A. Artuso, M. Lauriello and G. Coen Tirelli, "Pauci-Symptomatic Large Epidermoid Cyst of Cerebellopontine Angle: Case Report,” Acta Otorhinolaryngologica Italica, Vol. 24, No. 2, 2004, pp. 92-96. 